\title{
Quercetin-Loaded Lecithin/Chitosan Nanoparticles for Functional Food Applications
}

\author{
Marthyna P. Souza • Antônio F. M. Vaz • \\ Maria T. S. Correia $\cdot$ Miguel A. Cerqueira • \\ António A. Vicente • Maria G. Carneiro-da-Cunha
}

Received: 18 January 2013 / Accepted: 10 July 2013 / Published online: 4 August 2013

(C) Springer Science+Business Media New York 2013

\begin{abstract}
This study aimed at the encapsulation of quercetin into lecithin/chitosan nanoparticles using the electrostatic self-assembly technique, followed by evaluation of their functionality (antioxidant activity) and stability at different environmental conditions. These nanoparticles were characterized in terms of: average size, morphology, zeta potential, encapsulation efficiency, loading, and spectroscopic characteristics. Quercetin has been successfully encapsulated in lecithin/ chitosan nanoparticles with an efficiency of $96.13 \pm 0.44 \%$. Nanoparticles presented a spherical morphology with an average size of $168.58 \pm 20.94 \mathrm{~nm}$ and a zeta potential of $56.46 \pm 1.94 \mathrm{mV}$. Stability studies showed that nanoparticles are stable to temperatures ranging between 5 and $70{ }^{\circ} \mathrm{C}$ and a $\mathrm{pH}$ variation from 3.3 to 5.0. Moreover, encapsulated quercetin showed improved antioxidant properties when compared to free-quercetin. Our results suggest that quercetin-loaded lecithin/chitosan nanoparticles can be used in the manufacture of functional foods.
\end{abstract}

M. P. Souza $\cdot$ M. T. S. Correia $\cdot$ M. G. Carneiro-da-Cunha Biochemistry Department, Universidade Federal de Pernambuco-UFPE, Av. Prof. Moraes Rego s/n, CEP 50.670-420 Recife, PE, Brazil

M. P. Souza • M. A. Cerqueira • A. A. Vicente IBB - Institute for Biotechnology and Bioengineering, Centre of Biological Engineering, Universidade do Minho, Campus de Gualtar, 4710-057 Braga, Portugal

A. F. M. Vaz

Unidade Acadêmica de Medicina Veterinária, Universidade Federal de Campina Grande, CEP 58.7000-970 Patos, PB, Brazil

M. G. Carneiro-da-Cunha $(\bowtie)$

Laboratório de Imunopatologia Keizo Asami-LIKA/UFPE, Campus Universitário, s/n, CEP 50670-901 Recife, PE, Brazil

e-mail:mgcc@ufpe.br
Keywords Quercetin-loaded nanoparticles · Bioactive compounds encapsulation · Nanostructure self-assembly · Lecithin/chitosan nanoparticles $\cdot$ Antioxidant

\section{Introduction}

Nanotechnology has potential to produce new food ingredients and innovative products, with considerable benefits to human health (Magnuson et al. 2011). This can be achieved through the development of new structures for applications in functional foods (Huang et al. 2010). The consumption of foods providing health benefits as well as basic nutrition is very attractive to the consumer, and significant developments from the food industry have emerged in recent years.

Polyphenols typically used as part of the human diet are one of the most numerous and ubiquitous groups of plant metabolites that have a large spectrum of biological activities and may be considered chemopreventive agents in humans (Fang and Bhandari 2010). Quercetin $\left[3,3^{\prime}, 4^{\prime}, 5,7-\right.$ pentahydroxyflavone] is one of the most representative compounds of the flavonoid family, having been assigned a wide range of health benefits, including antioxidant activity, cancer prevention, protection to DNA damage, anti-inflammatory, and cardio-protective actions (An et al. 2010). However, quercetin is a molecule with a low bioavailability due to its low solubility, gastrointestinal instability, and low absorption in the gastrointestinal tract, which limits its biological effects in vivo (Chitkara et al. 2012). A good option to improve bioavailability of quercetin is nanoencapsulation that can increase the absorption in the gastrointestinal tract and enhance the molecule's stability against possible adverse conditions during processing, distribution, storage, and consumption of food products. Promising results, reported by Li et al. (2009), showed that when encapsulating quercetin in solid lipid nanoparticles, consisting of soy lecithin and glyceryl monostearate, the oral bioavailability in rats has doubled. An additional benefit is the 
increase of quercetin solubility. Quercetin encapsulation will allow its use as antioxidant in food products where solubility is a problem.

The oxidation of proteins and lipids is responsible for the development of unpleasant odors and flavors that make food unfit for consumption. These changes are not restricted to nutritional quality, due to the degradation of fat-soluble vitamins and essential fatty acids, but they also affect the integrity and safety of food through the formation of potentially toxic compounds (Ramalho and Jorge 2006). In this context, encapsulation of quercetin emerges as an alternative for the food industry to improve the defense against free radicals in various environments in a controlled manner.

Lecithin is a mixture of phospholipids of vegetable origin comprised mainly of phosphatidylcholine and phosphatidylethanolamine, and it is considered a safe and biocompatible excipient, already used in many pharmaceutical formulations (Şenyiğit et al. 2010). Chitosan is a cationic and linear heteropolysaccharide composed of units of $N$-acetylglucosamine (GlcNAc) and glucosamine (GlcN) linked by $\beta-1,4$ bonds. The quantity and sequence of these units determines the physical, chemical, and biological properties of the polymer (Prashanth and Tharanathan 2007). Chitosan, commonly obtained from the alkaline or enzymatic deacetylation of chitin (Shahidi and Abuzaytoun 2005) showed multiple properties such as biocompatibility, biodegradability, bioadhesion, absence of allergenicity and toxicity, anti-hypercholesterolemic, and antimicrobial activity; and has been presented as having potential for application in the industries of food, cosmetics, and pharmaceutics (Gerelli et al. 2008; Chuah et al. 2009; Şenyiğit et al. 2010). In particular, studies have shown that when used in optimum conditions of preparation (i.e., concentration, $\mathrm{pH}$, ionic strength, and electrophoretic mobility); chitosan provides structural stability to nanoemulsions and liposomes (Chuah et al. 2009; Panya et al. 2010).

Recently, lecithin and chitosan-based nanoparticles obtained from self-organized supramolecular electrostatic interactions between a positively charged polysaccharide and negatively charged lipids, have been described as a suitable carrier for hydrophobic molecules (Sonvico et al. 2006). Hafner et al. (2009) showed that melatonin-loaded lecithin/chitosan nanoparticles, when using Lipoid S45 as lecithin source, presented mucoadhesive properties, absence of cytotoxicity, and promoted the increase of permeability of melatonin against an in vitro model of epithelial barrier consisting of Caco-2 cells.

In the present study the encapsulation of quercetin into lecithin/chitosan nanoparticles is reported. Subsequently, their functionality is evaluated through antioxidant activity measurements and their stability is related to $\mathrm{pH}$, temperature, and storage time as essential parameters for the use of quercetin-loaded lecithin/chitosan nanoparticles by the food industry, either as part of functional foods or controlling harmful free radicals effects in foods.

\section{Material and Methods}

\section{Materials}

Soya lecithin (Lipoid S45) was purchased from Lipoid (Ludwigshafen, Germany), chitosan (90\% deacetylation, $\mathrm{MW}=3.4 \times 10^{5} \mathrm{Da}$ ) from Aqua Premier Co. Ltd (Thailand) and $99 \%$ ethanol from Panreac (Spain). The solvents used were of analytical grade and obtained from Merck (Darmstadt, Germany). All other reagents were purchased from Aldrich (Steinheim, Germany). Water was purified through a Millipore Q purification system (Millipore Corp., Bedford, MA).

\section{Nanoparticles Preparation}

Lecithin/chitosan nanoparticles were prepared according to the methodology described by Sonvico et al. (2006) with some modifications. The suspension of nanoparticles was obtained by dropwise injection (syringe having internal diameter of $0.38 \mathrm{~mm}, 1 \mathrm{~mL} \mathrm{~min}{ }^{-1}$ injection rate) of $2 \mathrm{~mL}$ of ethanolic solution of Lipoid S45 $(25 \mathrm{mg} / \mathrm{mL})$ in $23 \mathrm{~mL}$ aqueous solution of chitosan $(0.11 \mathrm{mg} / \mathrm{mL})$ under mechanical stirring $(24,000 \mathrm{rpm}$, TP 18/10-10N Ultraturrax, IKA Werke, Staufen, Germany). The chitosan aqueous solution was prepared by diluting a standard solution of $0.2 \%$ chitosan $(w / v)$ in lactic acid $(1 \% v / v)$. The incorporation of quercetin at different concentrations $(50-800 \mu \mathrm{g} / \mathrm{mL})$ was performed by its dissolution in ethanolic solution of lipoid S45. The lecithin/chitosan nanoparticles and quercetinloaded lecithin/chitosan nanoparticles were called $\mathrm{Np}$ and Quercetin-Np, respectively.

\section{Physical-Chemical Characterization}

\section{Particle Size and Zeta Potential Measurements}

The hydrodynamic diameter (size-average) and polydispersity index (PDI) of the nanoparticles were determined by dynamic light scattering (Zetasizer Nano ZS, Malvern Instruments, UK). The samples were diluted (1:3) with Milli-Q water (Milli-Q synthesis, $18.2 \mathrm{M} \Omega \mathrm{cm}$ ) and analyzed in a folded capillary cell. Detection of the scattered light was carried out at $173^{\circ}(\mathrm{NIBS}=$ non-invasive backscatter detection) to reduce the path length of the scattered light from the samples and to minimize the risk of multi-scattering at $25^{\circ} \mathrm{C}$. The zeta potential was measured by laser Doppler microelectrophoresis using a Zetasizer ZS Nano (Zetasizer Nano ZS, Malvern Instruments, UK). All measurements were 
carried out in triplicate with three readings for each of them. The results are given as mean \pm standard deviation of the nine values obtained.

\section{Quercetin Encapsulation Efficiency and Loading}

The quercetin loading and encapsulation efficiency of the $\mathrm{Np}$ were conducted according to the modified procedures previously described by Li et al. (2009). Encapsulated and nonencapsulated portions of quercetin from the Quercetin-Np suspension were separated using centrifugal filter devices (Amicon Ultra-0.5, Millipore ${ }^{\circledR}$ ) with centrifugation at $14,000 \mathrm{rpm}$ for $30 \mathrm{~min}$. The ultrafiltrate and nanoparticles resulting from centrifugation were diluted with absolute ethanol and quercetin content was measured by UV/Vis spectrophotometry (Jasco, Japan) at $373 \mathrm{~nm}$. The encapsulation efficiency of quercetin (EE) and quercetin-loading (L) were calculated by the following equations:

$\mathrm{EE}(\%)=\left(W_{\text {total }}-W_{\text {free }}\right) \times 100 / W_{\text {total }}$

$L(\%)=\left(W_{\text {total }}-W_{\text {free }}\right) \times 100 / W_{\text {np }}$

where $W_{\text {total }}$ was the total quercetin weight in nanoparticles suspension; $W_{\text {free }}$ was the weight of free-quercetin in nanoparticles suspension; and $W_{\mathrm{np}}$ was the vehicle weight.

\section{Nanoparticles Morphology}

The morphology of Quercetin-Np was observed by transmission electron microscopy (EM 902nd, Zeiss, West Germany). The sample was diluted (1:3) with Milli-Q water and immobilized on copper grids for observation.

\section{UV/Vis Spectroscopy}

The UV-Vis absorption spectra of Quercetin-NP and freequercetin were obtained in a spectrophotometer (Jasco, Japan) in the range of 200-700 $\mathrm{nm}$ at $1.0 \mathrm{~nm}$ intervals. Samples were measured at $25^{\circ} \mathrm{C}$ in a rectangular quartz cuvette with $1 \mathrm{~cm}$ path length. All determinations were performed in triplicate.

\section{Fluorescence Spectroscopy}

The intrinsic fluorescence spectra of Quercetin-NP and freequercetin between 350 and $650 \mathrm{~nm}$ were determined with a fluorescence spectrophotometer (JASCO FP-6300, Tokyo, Japan). The excitation and emission bands were set at $2.5 \mathrm{~nm}$ and excitation wavelength at $617 \mathrm{~nm}$. All determinations were performed in triplicate.

\section{Fourier Transform Infrared Spectroscopy}

Fourier transform infrared (FTIR) spectra of Quercetin$\mathrm{Np}$ and free-quercetin were obtained by an infrared spectrometer (PerkinElmer 16 PC spectrometer, Boston, USA). The pre-lyophilized samples were ground with spectroscopic grade potassium bromide $(\mathrm{KBr})$ powder and then pressed into $1 \mathrm{~mm}$ pellet for FTIR measurement in the range of $450-4,000 \mathrm{~cm}^{-1}$ with $4 \mathrm{~cm}^{-1}$ resolution, using 16 scans.

Antioxidant Activities Assays

\section{DPPH Scavenging Capacity}

The free radical scavenging capacity of free-quercetin, $\mathrm{Np}$, and Quercetin-Np was measured by 1,1-diphenyl-2-picrylhydrazyl (DPPH) assay reported by Kumari et al. (2010) with modifications. Briefly, $20 \mu \mathrm{L}$ of sample solution was added into $200 \mu \mathrm{L}$ of ethanolic solution of DPPH $(200 \mu \mathrm{M})$ and the mixture was then vortexed vigorously and left for $30 \mathrm{~min}$ at room temperature in the dark. The absorbance of the reaction solution was measured at $515 \mathrm{~nm}$ (ELISA reader; Bio-Rad).

The percentage of scavenging of free radicals by the sample was calculated according to the following equation:

$$
\begin{aligned}
\text { Scavenging capacity }(\%)= & \left(1-\mathrm{Abs}_{515} \text { sample }\right) \\
& \times 100 / \mathrm{Abs}_{515} \text { control }
\end{aligned}
$$

All determinations were performed in triplicate and results are given as the mean \pm standard deviation.

\section{Reducing Power}

The test ferric reducing antioxidant power (FRAP) was performed according to Benzie and Strain (1996) with some modifications. Initially, $10 \mu \mathrm{L}$ of sample (free-quercetin, $\mathrm{Np}$, Quercetin-Np) were mixed with $290 \mu \mathrm{L}$ of FRAP reagent. The mixture was subsequently incubated at $37^{\circ} \mathrm{C}$ for $15 \mathrm{~min}$ and the absorbance determined at $593 \mathrm{~nm}$ (ELISA reader; Bio-Rad). A calibration curve was prepared with $\mathrm{FeSO}_{4} \cdot 7 \mathrm{H}_{2} \mathrm{O}$ as standard, in the range of $50-1,500 \mu \mathrm{M}$. FRAP reagent was freshly prepared by mixing $10 \mathrm{mM}$ 2,4,6-tris (1-pyridyl)-5triazine in $40 \mathrm{mM} \mathrm{HCl}$ with a $20 \mathrm{mM} \mathrm{FeCl}_{3}$ solution and $0.3 \mathrm{M}$ acetate buffer (pH 3.6) in the proportion 1:1:10 $(v / v / v)$. The results were expressed as micromoles equivalent $\mathrm{Fe}^{2+}\left(\mathrm{Fe}^{2+} \mu \mathrm{M}\right)$.

\section{Anti-lipid Peroxidation Assay}

The anti-lipid peroxidation effect of free-quercetin, $\mathrm{Np}$, and Quercetin-Np on $\mathrm{FeCl}_{2}$-ascorbic acid-induced lipid 
peroxidation in rat liver homogenate was carried out according to $\mathrm{Wu}$ et al. (2008). The $20 \%$ liver homogenate $(w / v)$ was prepared with $150 \mathrm{mM}$ Tris- $\mathrm{HCl}$ buffered saline solution $(\mathrm{pH} 7.2)$ and further centrifuged at $500 \times \mathrm{g}$ for $10 \mathrm{~min}$. Then $50 \mu \mathrm{L}$ of the homogenate were mixed with $30 \mu \mathrm{L}$ of the sample, $10 \mu \mathrm{L}$ of $4 \mathrm{mM} \mathrm{FeCl}_{2}$, and $10 \mu \mathrm{L}$ of $0.2 \mathrm{mM}$ ascorbic acid. The mixture was incubated at $37{ }^{\circ} \mathrm{C}$ for $1 \mathrm{~h}$, followed by the addition of $100 \mu \mathrm{L}$ of $0.1 \mathrm{M} \mathrm{HCl}$, $40 \mu \mathrm{L}$ of $9.8 \%$ SDS, $180 \mu \mathrm{L}$ of deionized water, and $400 \mu \mathrm{L}$ of $0.6 \%$ TBA to each tube. Tubes were vigorously shaken and placed at $100^{\circ} \mathrm{C}$ for $30 \mathrm{~min}$. After cooling, $1,000 \mu \mathrm{L} \mathrm{n}$ butanol was added to the tubes and centrifuged at $1,000 \times \mathrm{g}$ for $25 \mathrm{~min}$; the supernatant was collected and the absorbance measured at $532 \mathrm{~nm}$ (ELISA reader; Bio-Rad). All determinations were performed in triplicate and results are expressed as mean \pm standard deviation.

The percentage of lipid peroxidation was determined using the following equation:

$$
\begin{aligned}
& \% \text { peroxidation }=\left(\mathrm{Abs}_{532} \text { induced }-\mathrm{Abs}_{532 \text { sample }}\right) \\
& \times 100 /\left(\mathrm{Abs}_{532} \text { induced }-\mathrm{Abs}_{532} \text { control }\right)
\end{aligned}
$$

where $\mathrm{Abs}_{532}$ induced is the absorbance of a reaction in which $100 \%$ of lipid peroxidation occurred (containing distilled water instead of test sample); $\mathrm{Abs}_{532}$ sample is the absorbance of the samples (free-quercetin, $\mathrm{Np}$, and Quercetin-Np); $\mathrm{Abs}_{532}$ control is the absorbance of control reaction in the absence of rat liver homogenate.

Stability Studies of Quercetin-Np

\section{Effect of $p H$}

The effect of $\mathrm{pH}$ on the stability of Quercetin-Np was evaluated adjusting the $\mathrm{pH}$ of the samples from 3.5 to 8.0 (using $1 \mathrm{M} \mathrm{NaOH}$ ). The samples were stored at room temperature $\left(25{ }^{\circ} \mathrm{C}\right)$ and left overnight for analysis of hydrodynamic diameter and zeta potential.

\section{Effect of Temperature}

The temperature effect in Quercetin-Np stability was analyzed in the Zetasizer Nano ZS (Malvern Instruments, UK), which allows scanning the temperature while measuring particle size and polydispersity. The cell used for readings was a $12-\mathrm{mm}$ square glass cell, with circular aperture and stopper and temperatures varied from 5 to $70{ }^{\circ} \mathrm{C}$. The samples were cooled at $5{ }^{\circ} \mathrm{C}$ for $1 \mathrm{~h}$ and the reading was performed at that temperature. Subsequently, the samples were gradually heated up to $70{ }^{\circ} \mathrm{C}$, being always maintained for $1 \mathrm{~h}$ at predetermined temperatures $(5,10,15,20,25,30$, $35,40,45,50,55,60,65$, and $70{ }^{\circ} \mathrm{C}$ ) for analysis. Each measurement was performed in triplicate and the results expressed as mean \pm standard deviation.

\section{Effect of Storage Time}

The Quercetin-Np was stored at 4 and $30{ }^{\circ} \mathrm{C}$ for 90 days in the dark. The stability was determined by analysis of the hydrodynamic diameter, quercetin concentration, and DPPH scavenging capacity. To determine quercetin concentration an aliquot of Quercetin-NP suspension was diluted with absolute ethanol and the absorbance measured spectrophotometrically at $373 \mathrm{~nm}$ using quercetin as standard, in the range of $1-50 \mu \mathrm{g} / \mathrm{mL}$.

DPPH scavenging capacity assays were performed by adding $1 \mathrm{~mL}$ of a DPPH stock solution $(100 \mu \mathrm{M}$ in methanol) to $100 \mu \mathrm{L}$ of Quercetin-Np suspension. The mixture was incubated in the dark under stirring for $30 \mathrm{~min}$ and absorbance was measured at $515 \mathrm{~nm}$ in a spectrophotometer, at $25{ }^{\circ} \mathrm{C}$ in a rectangular quartz cuvette with a $1-\mathrm{cm}$ path length. DPPH concentration was expressed as $\mu \mathrm{M}\left(\varepsilon=11.240 \mathrm{~mol} \mathrm{~L}^{-1} \mathrm{~cm}^{-1}\right.$ Portes et al. 2009). DPPH radical degrades spontaneously making it impossible to use the same initial solution when the experiment is performed for long periods of time (days). To reduce the fluctuations during preparation of the initial DPPH solution, we decided to calculate the concentration of the solution using the molar extinction coefficient on a specific solvent (in this case methanol), which is the reason why a different method for DPPH was used at this stage of the work.

All analyzes were carried out in triplicate and results were expressed as mean \pm standard deviation.

\section{Statistical Analysis}

All results were expressed as mean \pm standard deviation, and analyzed with one-way analysis of variance followed by a multi-parametric Tukey's post hoc test in GraphPrism ${ }^{\circledR}$ (GraphPad Software Inc., San Diego, CA). Statistical significance was established at $p<0.01$.

\section{Results and Discussion}

Nanoparticles Characterization

\section{Particles Size}

The size and polydispersity index are essential analyses for characterization of nanoparticles, since they influence important parameters such as loading, release, and stability of the compound inside nanoparticles. It is known that the smaller the particle the greater is the exposed surface area, which leads to a faster release of encapsulated drugs. Smaller 
particles also have an increased risk of aggregation during storage, being important the development of nanoparticles with a low PDI to achieve maximum stability by a better control (and lesser dispersion) of particles' size. It is worth mentioning that the reproducibility of parameters such as stability and release is directly connected to a low PDI $(\leq 0.4)$, since a high PDI means that there is no uniformity in the size distribution of the sample. Figure 1a shows that increasing quercetin concentrations in Quercetin-Np formulations lead to higher values of average size and polydispersity index. The formulations containing $0,50,70$, and $100 \mu \mathrm{g}$ of quercetin/mL suspension were homogeneous with low polydispersity index $(\mathrm{PDI} \leq 0.34)$ and average sizes of $117.7 \pm 5.73,133.25 \pm 8.91,168.58 \pm 20.94$, and $172.06 \pm 5$, respectively. Results are in agreement with the works published by Ghosh et al. (2011) and Pool et al. (2012) that when encapsulating quercetin in poly(lactic-co-glycolic acid) (PLGA) nanoparticles obtained particle sizes of $13.28 \pm 7.8 \mathrm{~nm}$ and $399.67 \pm 10.86 \mathrm{~nm}$, respectively. When the nanoparticles were prepared with values higher than $100 \mu \mathrm{g}$ quercetin $/ \mathrm{mL}$ nanoparticle aggregation was observed a few minutes after production. This behavior can be explained by the presence of high concentrations of quercetin that will interfere with the process of self-organization of nanoparticles by blocking the repulsive forces between the nanoparticles, due to the decrease of spacing between adjacent nanoparticles, thus leading to aggregation. A similar behavior was observed by Barbieri et al. (2013) for lecithin/chitosan nanoparticles with high loading of tamoxifen citrate. Based on these results only Quercetin-Np with concentrations lower than $100 \mu \mathrm{g}$ of quercetin $/ \mathrm{mL}$ were further evaluated.

\section{Quercetin Loading and Encapsulation Efficiency}

Loading and encapsulation efficiency depends largely on the compound solubility in the matrix material or polymer (dissolution or solid dispersal) (Kumari et al. 2010). The encapsulation efficiency of quercetin into lecithin/chitosan nanoparticles (Fig. 1b) presented values higher than $95 \%$ for all used concentrations $(50,70$ and $100 \mu \mathrm{g} / \mathrm{mL})(p<0.01)$. These efficiencies are higher than those obtained by Ghosh et al. (2011) and Pool et al. (2012) for PLGA nanoparticles (encapsulation efficiency below $80 \%$ ). The high encapsulation efficiency obtained with lecithin/chitosan nanoparticles may be related to the fact that both lecithin and quercetin are hydrophobic molecules, which allows a strong affinity between them. Consequently, a larger amount of quercetin was preferably inserted into the lipid nuclei of the nanoparticles, and only a small amount of quercetin was lost in the aqueous phase during the preparation process. This loss is in agreement with the value of solubility for quercetin in water $(0.514 \mu \mathrm{g} / \mathrm{mL})$ (Saija et al. 2003). Other molecules with hydrophobic character such as progesterone, melatonin, and clobetasol-17-propionate were also successfully encapsulated in lecithin/chitosan nanoparticles (Hafner et al. 2009; Sonvico et al. 2006; Șenyiğit et al. 2010).

Figure $1 \mathrm{~b}$ also shows that increasing the quercetin concentration in the formulation resulted in improved quercetin loading $(p<0.01)$. However, formulations containing $100 \mu \mathrm{g} / \mathrm{mL}$ of quercetin showed signs of instability (precipitation) after 1 week; therefore, nanoparticles with $70 \mu \mathrm{g} / \mathrm{mL}$ of quercetin were chosen to be used in further analyses.

\section{Zeta Potential}

Zeta potential represents the surface charge that can influence the stability of suspended particles through electrostatic repulsion among them. Quercetin-Np showed zeta potential values of $56.46 \pm 1.94 \mathrm{mV}$. This value is suitable for forming a stable suspension of nanoparticles, since particles can be dispersed stably when the absolute value of zeta potential is above $30 \mathrm{mV}$ (Liu et al. 2007). At the original $\mathrm{pH}$ of Quercetin-Np ( $\mathrm{pH} 3.3)$, the phosphate groups $\left(\mathrm{PO}_{3}^{-}\right)$of lecithin $(\mathrm{pKa} \sim 1.5)$ and the amino groups $\left(\mathrm{NH}_{3}{ }^{+}\right)$of chitosan
Fig. 1 a Average particle size (black squares) and polydispersity index (white squares) versus quercetin concentration. b Influence of quercetin concentration in the formulation, loading (white squares) and encapsulation efficiency (black diamonds) of $\mathrm{Np}$. All determinations were performed in triplicate and the results expressed as mean \pm standard deviation
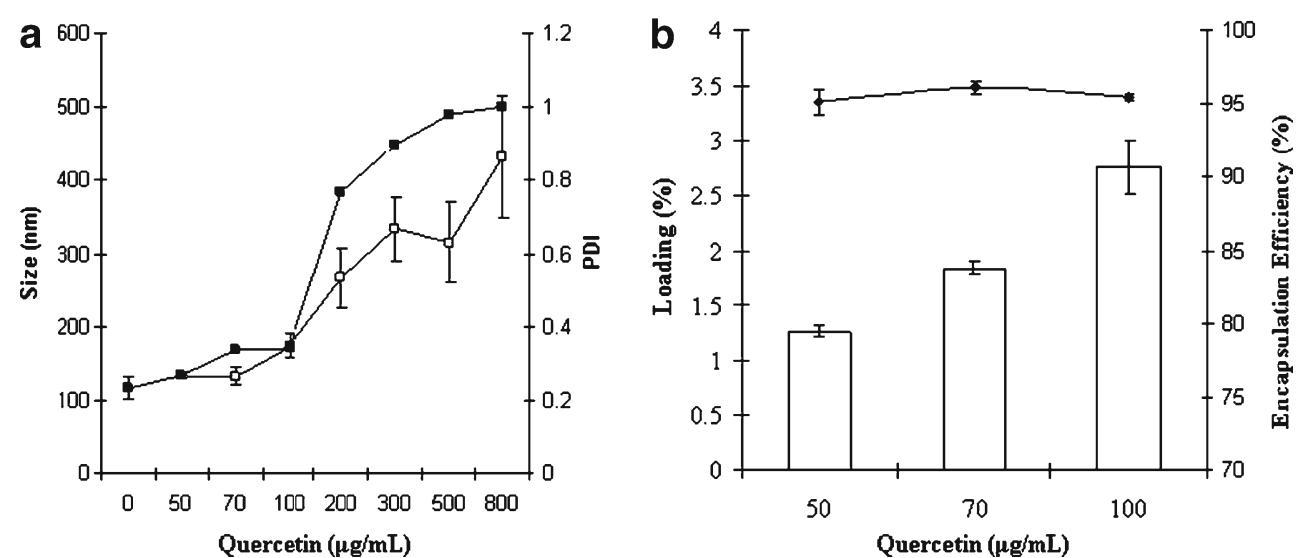
(pKa 6.2-7.0) have opposing charges; it is thus presumed that a protective chitosan layer around the particles of Quercetin-Np is formed through electrostatic interactions (Chuah et al. 2009). Thus, the amino groups of chitosan

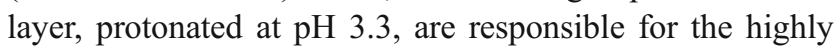
positive zeta potential of nanoparticles. When assessing chitosan solutions with $\mathrm{pH}$ values between 2.5 and 3.5, Carneiro-da-Cunha et al. (2011) showed that zeta potential can vary between 31.6 and $63.5 \mathrm{mV}$.

\section{Nanoparticle Morphology}

Transmission electron micrographs of Quercetin-Np are shown in Fig. 2. Figure 2a shows that the nanoparticles are spherical and polydisperse, with sizes ranging from 60 to $500 \mathrm{~nm}$. Figure $2 \mathrm{~b}$ shows a compact lipid nuclei surrounded by a contrasted layer of chitosan which confirms its presence as the outmost layer surrounding the quercetin-lecithin complex, as the result of expected electrostatic interaction based on oppositely charges (zeta potential) of solutions previously measured, in order to support the assembly.

\section{Spectroscopy Evaluation}

Figure 3a shows UV-Vis spectra of free-quercetin (spectrum a) and-Quercetin-Np (spectrum b). The spectrum of freequercetin shows two absorption bands, at 257 and $373 \mathrm{~nm}$, related to conjugations in the B-ring and A-ring, respectively, similar to those described by Timbola et al. (2006). Quercetin-Np showed a characteristic peak of turbidity, which is indicative of nanoparticles formation. A similar profile was obtained by Kumari et al. (2010) for PLA (Poly-D, L-lactide) nanoparticles. As well as for free-quercetin, the spectrum of Quercetin-Np also showed absorption bands at 257 and $373 \mathrm{~nm}$, confirming the presence of quercetin in the Np's.

Quercetin is slightly fluorescent and extremely sensitive to changes that may occur in the medium, such as polarity and hydrogen bonding effects. Thus, its encapsulation in $\mathrm{Np}$ caused increased fluorescence emission intensity (Fig. 3b) compared with free-quercetin, indicating changes in the microenvironment. This is probably because the Np offers a less polar and more rigid protective microenvironment shielding the excited species from quenching and nonradioactive decay processes. Similar results were obtained with the encapsulation of quercetin into chitosan nanoparticles (Zhang et al. 2008).

FTIR analyses were performed to investigate a possible reaction between quercetin and $\mathrm{Np}$. The FTIR spectrum obtained for free quercetin (Fig. 3c) showed bands and typical molecular peaks of its structure: $1,381 \mathrm{~cm}^{-1}(\mathrm{C}-$ $\mathrm{OH}), 1,610 \mathrm{~cm}^{-1}(\mathrm{C}=\mathrm{C}), 1,264 \mathrm{~cm}^{-1}(\mathrm{C}-\mathrm{O}-\mathrm{C}), 1,662 \mathrm{~cm}^{-1}$ $(\mathrm{C}=\mathrm{O})$, and $3,403 \mathrm{~cm}^{-1}(\mathrm{O}-\mathrm{H}$ stretch). The $\mathrm{Np}$ spectrum (Fig. 3c) showed characteristic chitosan bands with peaks at 1,650 and $1,590 \mathrm{~cm}^{-1}$ attributed to NH-bending units of glucosamine. The absorption bands at $1,051 \mathrm{~cm}^{-1}(\mathrm{C}-\mathrm{O}-$ C) and $1,080 \mathrm{~cm}^{-1}$ (skeletal vibration involving the $\mathrm{C}-\mathrm{O}$ stretching) are characteristic of its saccharide structure. The presence of an absorption band at $1,217 \mathrm{~cm}^{-1}$ corresponding to $\mathrm{P}=\mathrm{O}$ stretching indicates an ionic interaction between the phosphate groups of lecithin and the amino group of chitosan. No characteristic peaks of quercetin were observed in the spectrum of Quercetin-Np, which may be indicative of quercetin encapsulation in the $\mathrm{Np}$, and this interaction probably occurred by hydrophobic interactions or hydrogen bonds. The disappearance of characteristic peaks of quercetin after encapsulation was also observed in chitosan nanoparticles by Zhang et al. (2008).

\section{Antioxidant Activity Evaluation}

The DPPH scavenging capacity assay was used to evaluate the ability of free-quercetin, Quercetin-Np, and Np to donate protons. Figure 4a shows that the capacity of the phenolic group of quercetin to donate hydrogen in order to stabilize free radicals was maintained after encapsulation. Quercetin$\mathrm{Np}$ were capable to reduce higher numbers of DPPH molecules when compared with free-quercetin $(p<0.01)$, which can be related with improved dissolution properties of quercetin after encapsulation. This phenomenon was also
Fig. 2 Transmission electron microscopy images of: a morphology and dispersivity of Quercetin-Np; b compact core of quercetin-lecithin complex surrounded by a transparent layer of chitosan
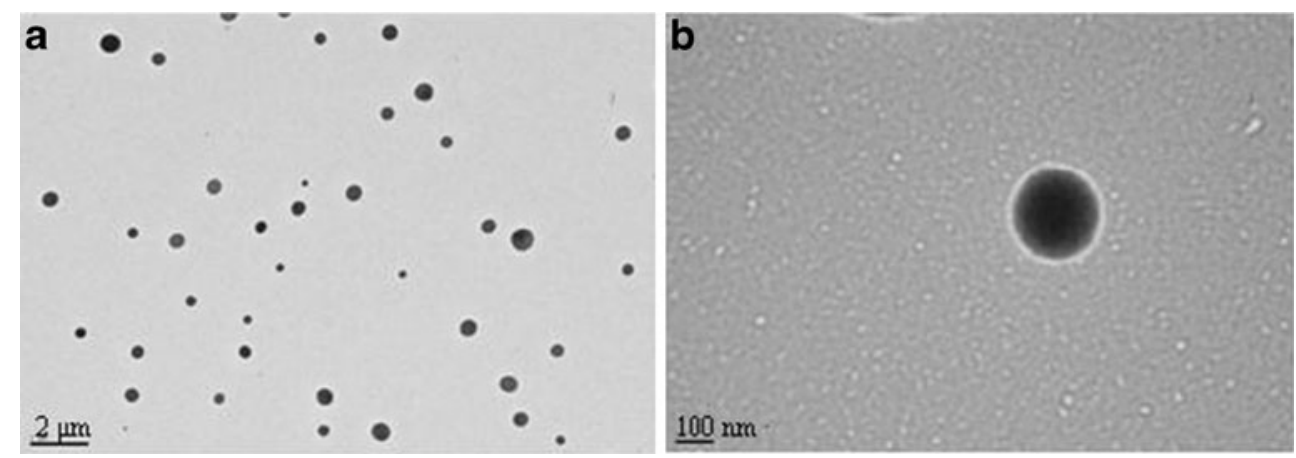
Fig. 3 a UV-Vis spectra of freequercetin $(a)$ and Quercetin-Np (b). b Fluorescence emission spectra of equimolar $a$ encapsulated and $b$ freequercetin molecule. c FTIR spectra of free-quercetin $(a)$ and Quercetin-Np $(b)$

\section{a}

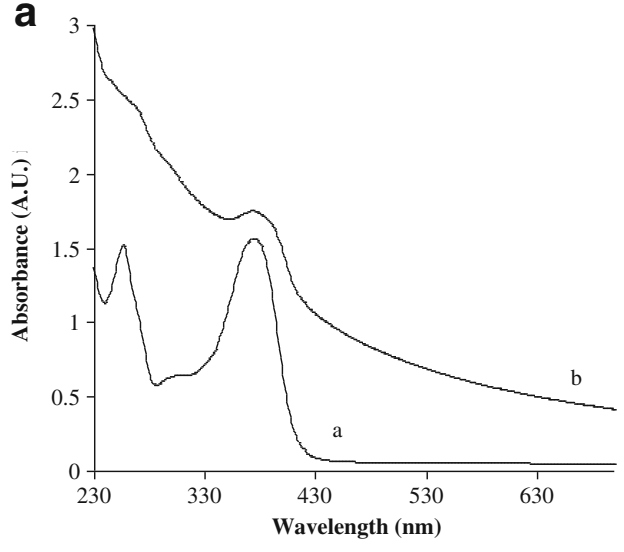

b

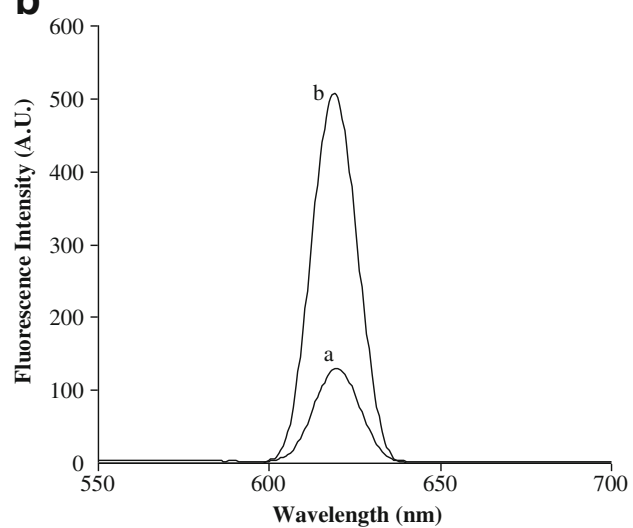

C

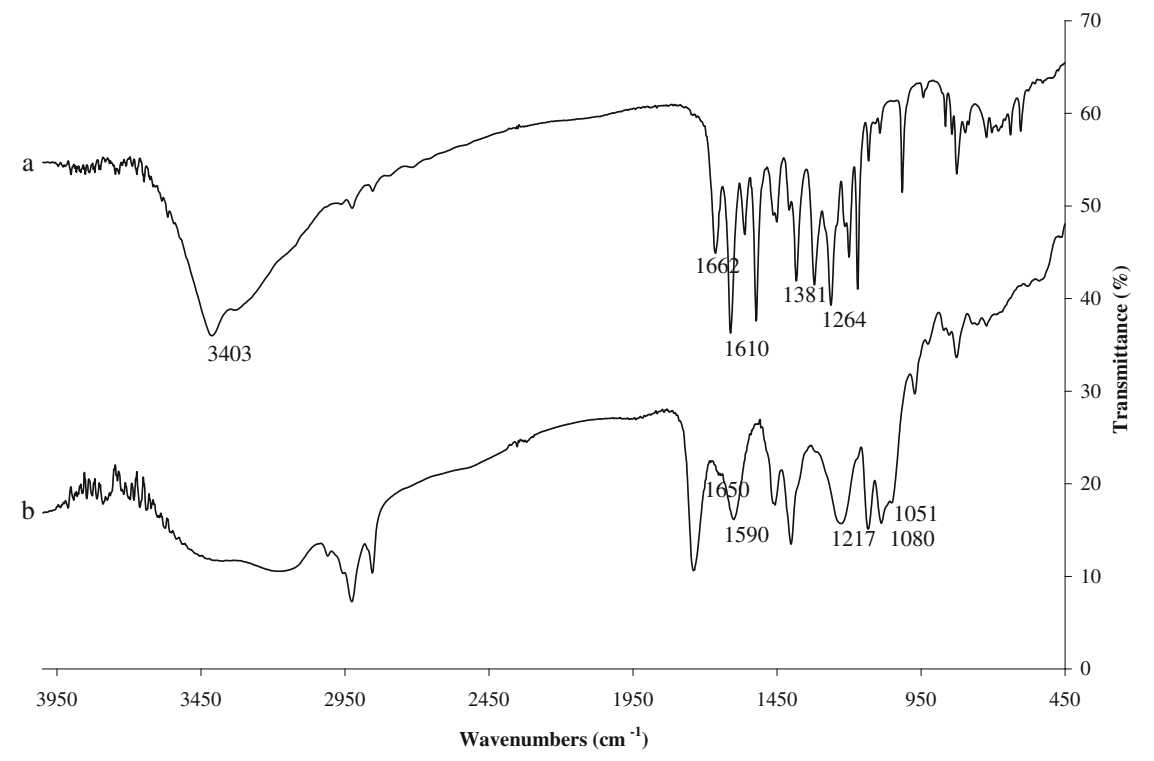

observed by Wu et al. (2008) when encapsulating quercetin in nanoparticles based on polyvinyl alcohol and Eudragit ${ }^{\circledR}$.

The reducing power of a compound can serve as a significant indicator of its antioxidant capacity (Firuzi et al. 2005). Figure $4 \mathrm{~b}$ shows the reducing power of free quercetin, Quercetin-Np, and Np. Quercetin-Np were more effective in reducing $\mathrm{Fe}^{3+}$ to $\mathrm{Fe}^{2+}$ in comparison with free-quercetin $(p<0.01)$. This property of nanoparticles is entrusted exclusively to the ability to donate protons of the phenolic groups of quercetin, since the $\mathrm{Np}$ were not able to reduce $\mathrm{Fe}^{3+}$. The increased reducing power of quercetin after encapsulation again can be probably explained by an improvement in the dissolution properties of quercetin in aqueous medium.

The oxidation process of food products during production and storage causes a sequence of particularly unfavorable changes in the sensory properties of the product (appearance of rancidity, changes in color and texture) responsible for quality downgrading and economic losses (Gramza and Korczar 2005). An assay based on inhibition of stimulated lipid peroxidation in rat liver homogenate by a system of ferrous ion-vitamin $\mathrm{C}$ was used to evaluate the protective effect of Quercetin-Np against lipid peroxidation. Figure 4c shows the percentage of peroxidation using as standard a reaction where there is $100 \%$ peroxidation of lipids present. It is observed that systems containing free-quercetin and Quercetin-Np showed only 8.23 and $17.62 \%$ of lipid peroxidation, respectively, leading to protection levels of approximately $91 \%$ (free-quercetin) and $83 \%$ (Quercetin-Np), demonstrating the efficiency of the system of Quercetin$\mathrm{Np}$. A rate of $235 \%$ for peroxidated lipids in the system containing $\mathrm{Np}$ can be explained by the fact that nanoparticles are mainly composed of lecithin which is also subject to peroxidation by the ferrous ion-vitamin $\mathrm{C}$ system used.

Evaluation of Quercetin-Np Stability

\section{Effect of pH Changes}

The foods' $\mathrm{pH}$ may range from acidic (e.g., from 2.5 to 4 , in some soft drinks) to slightly alkaline (e.g., from 7 to 7.4 , in 

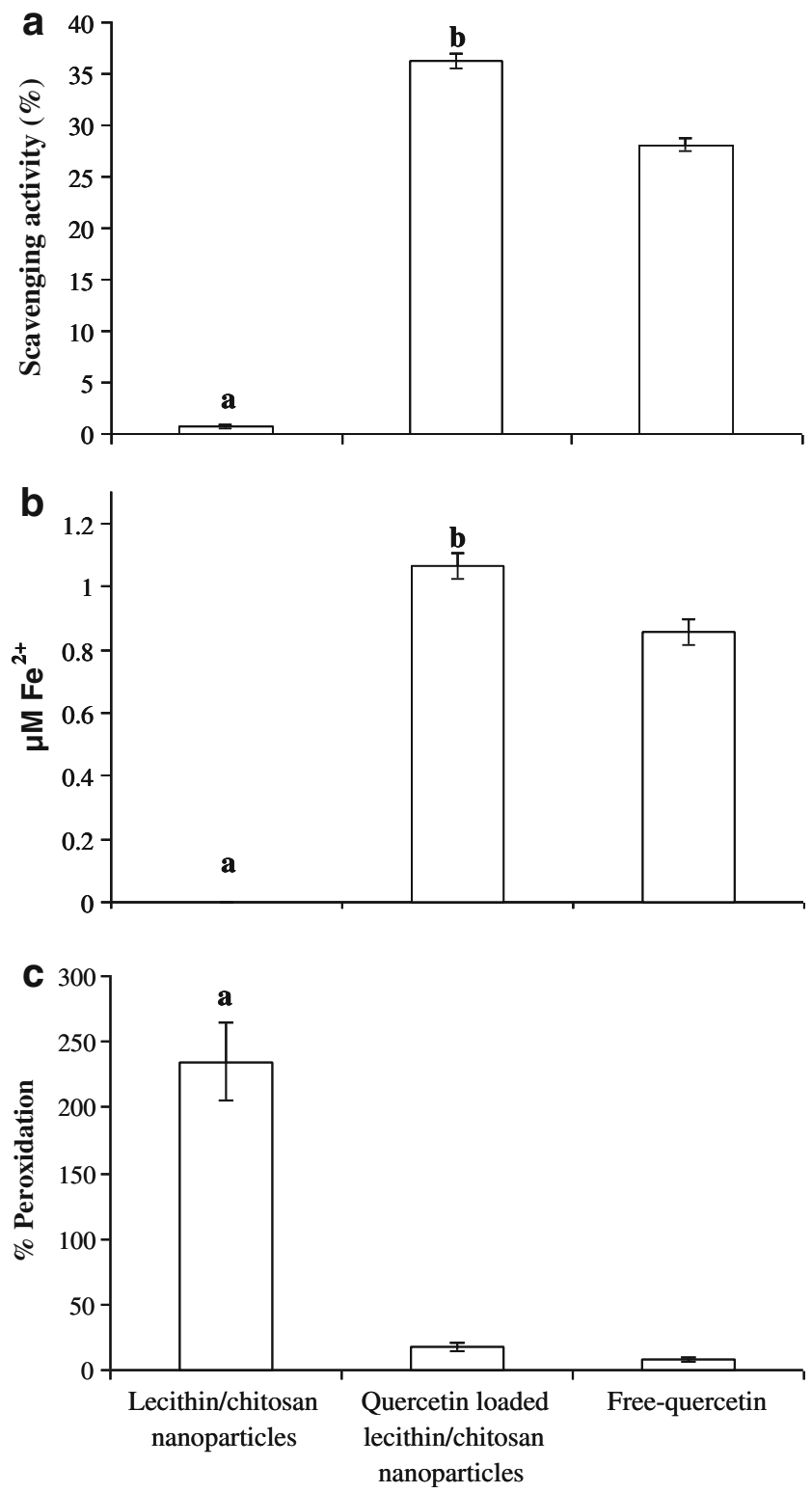

Fig. 4 Antioxidant activity of $\mathrm{Np}$, Quercetin-Np, and free-quercetin evaluated by: DPPH scavenging capacity (a), reducing power (FRAP) (b), and anti-lipid peroxidation assay (c). All determinations were performed in triplicate and the results expressed as mean \pm standard deviation. $a$ Significantly different from the Quercetin-Np and freequercetin at $p<0.01 ; b$ significantly different from free-quercetin at $p<0.01$

some dairy products), depending on the nature of the product. In addition, $\mathrm{pH}$ can vary during production, storage, and consumption. Medium $\mathrm{pH}$ determines the ionization of groups present on the surfaces of nanoparticles and, consequently, their surface final loading density (Heurtault et al. 2003). Thus, it is very important to ensure that Quercetin-Np remain stable when exposed to $\mathrm{pH}$ changes.

Figure 5a shows the effect of $\mathrm{pH}$ variation on zeta potential of Quercetin-Np. As pH was raised from 3.3 to 6.5, the electrical charge of the particles was decreasing and became negative for values above $\mathrm{pH}$ 7.0. The density reduction of positive charges in Quercetin- $\mathrm{Np}$ with $\mathrm{pH}$ increase is possibly the result from deprotonation of amino groups present in chitosan, whose $\mathrm{pKa}$ is about 6.2-7.0. As chitosan loses its positive charge, electrostatic attraction between the anionic lecithin molecules and the cationic chitosan molecules decreases. Therefore, it is possible that chitosan molecules have dissociated from the surface of Quercetin-Np, which would explain the observed negative zeta potential at alkaline $\mathrm{pH}$ values.

Variations in surface charge of Quercetin-Np, resulting from the increase of $\mathrm{pH}$ values, significantly influence their stability in solution (Fig. 5b). The average size of QuercetinNp remained constant up to $\mathrm{pH} 5.0$ with signs of aggregation at $\mathrm{pH} 5.5$ and later flocculation above $\mathrm{pH}$ 6.5. For $\mathrm{pH}$ values between 3.3 and 5.0 the Quercetin-Np is highly positive which generates a strong electrostatic repulsion. As already mentioned, as the solution $\mathrm{pH}$ was increased, chitosan molecules responsible for the high density of positive charges in Quercetin-Np are deprotonated and consequently the repulsion between the Quercetin-Np also decreased leading to an aggregation ( $\mathrm{pH}$ 5.5-6.0). The $\mathrm{pH}$ range where a higher degree of flocculation of Quercetin-Np was observed occurred between 6.5 and 7.0, which corresponds to the pKa region of chitosan. This behavior of flocculation and aggregation in the $\mathrm{pH}$ range close to the $\mathrm{pKa}$ of chitosan suggests that Quercetin-Np stability is predominantly governed by the presence of this polysaccharide. The flocculation observed may be explained by partial desorption of chitosan molecules from the surface of Quercetin-Np, leading to formation of polymeric bonds between chains. Quercetin- $\mathrm{Np}$ at $\mathrm{pH} 8.0$ became more stable because chitosan molecules lost most of their positive charge and are therefore not effective to induce flocculation. Moreover, lecithin zeta potential is constant above $\mathrm{pH} 2.0$ and contributes to the overall charge of Np's. Above $\mathrm{pH} 8.0$ chitosan chains are deprotonated and Np's charge will be dominated by lecithin charge. Similar behavior was observed by Chuah et al. (2009) in emulsions of soy lecithin coated with chitosan.

\section{Effect of Temperature Variation}

The resistance of Np's to temperature changes is of great importance for food applications, once some foods undergo some type of thermal processing during production, storage, or consumption, such as pasteurization, sterilization, or cooking (Guzey and McClements 2006).

Quercetin-Np showed to be stable under thermal treatment in the range $5-70{ }^{\circ} \mathrm{C}$ (Fig. 6a). The coalescence phenomenon that normally occurs in lipid systems was not observed, probably due to the protective layer of chitosan, with high density of positive charges, which generates electrostatic repulsion between particles. Chuah et al. (2009) 
Fig. 5 a Effect of $\mathrm{pH}$ variation on zeta potential of Quercetin$\mathrm{Np}$; b effect of $\mathrm{pH}$ variation on the average size of Quercetin$\mathrm{Np}$. All determinations were performed in triplicate and the results expressed as mean \pm standard deviation

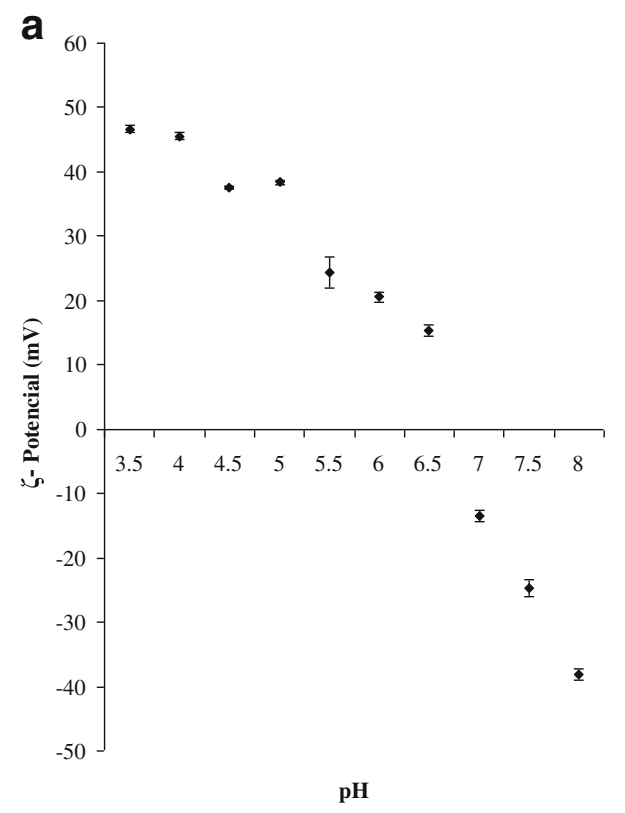

when assessing the thermal treatment effect $\left(30-90{ }^{\circ} \mathrm{C}\right)$ in lecithin nanoemulsions found that addition of chitosan to these systems makes them more stable to temperature variations. The slight reductions in average size and PDI observed in Quercetin-Np are probably a result of disaggregation of particles caused by agitation of molecules with the energy input into the system when temperature is raised.

\section{Stability During Storage Time}

Np's can be considered stable when there is no perceptible change in the particle size distribution, in the state of aggregation or in the spatial arrangement within the colloidal

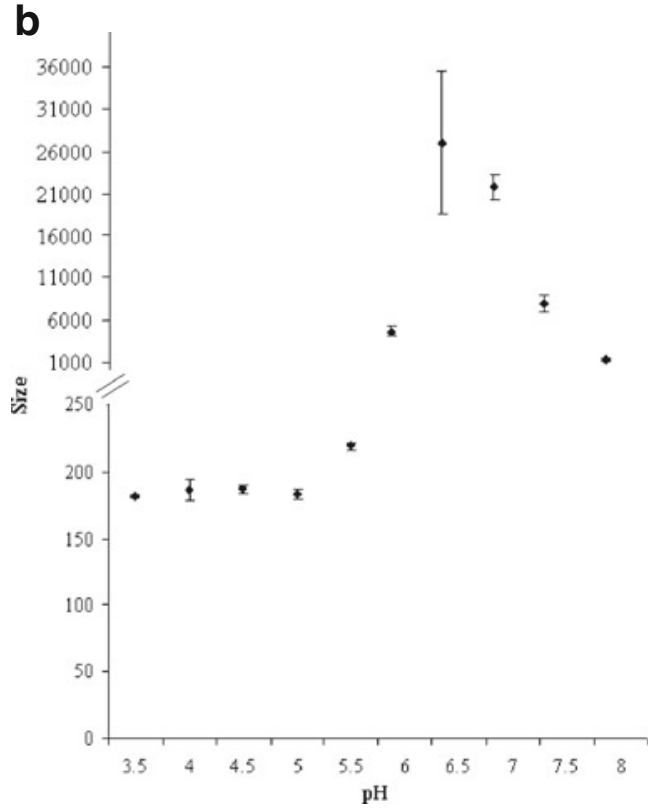

system over a period of observation (Dickinson 2003). Quercetin-Np stability was evaluated during 90 days at $4{ }^{\circ} \mathrm{C}$ and $30{ }^{\circ} \mathrm{C}$. No statistically significant differences $(p<0.01)$ were observed in particle size, PDI, and zeta potential (data not shown). The same behavior was reported for melatonin-loaded lecithin/chitosan nanoparticles stored at $4{ }^{\circ} \mathrm{C}$ for 60 days, for average size and zeta potential (Hafner et al. 2009).

Figure $6 \mathrm{~b}$ shows the variation of quercetin concentration and antioxidant activity (DPPH scavenging capacity) during Quercetin-Np storage at 4 and $30{ }^{\circ} \mathrm{C}$. The Quercetin-Np stored at $30^{\circ} \mathrm{C}$ showed gradual and linear reduction in DPPH consumption at the sixth day of storage combined with the

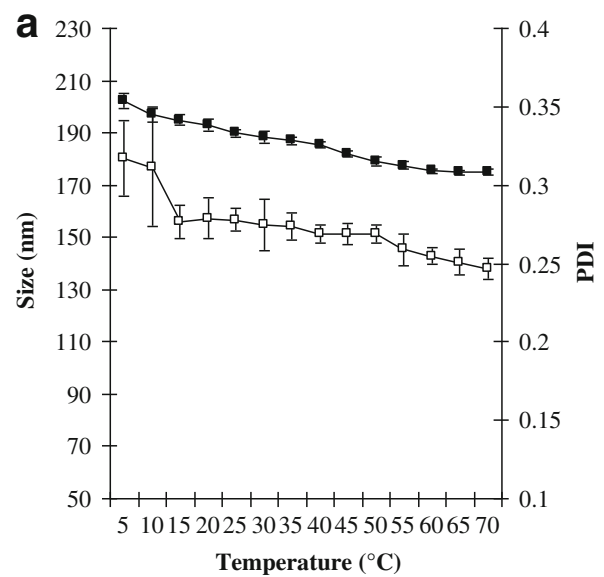

Fig. 6 a Effect of temperature variation in the average size (black squares) and polydispersity index (white squares) of Quercetin-Np. b Effect of storage time in quercetin concentration $(\mu \mathrm{g} / \mathrm{mL})$ and DPPH scavenging capacity ( $\mu \mathrm{mol}$ ) of Quercetin-Np stored at 4 and $30{ }^{\circ} \mathrm{C}$. Key: whites circles concentration of Quercetin-Np stored at $4{ }^{\circ} \mathrm{C}$, black

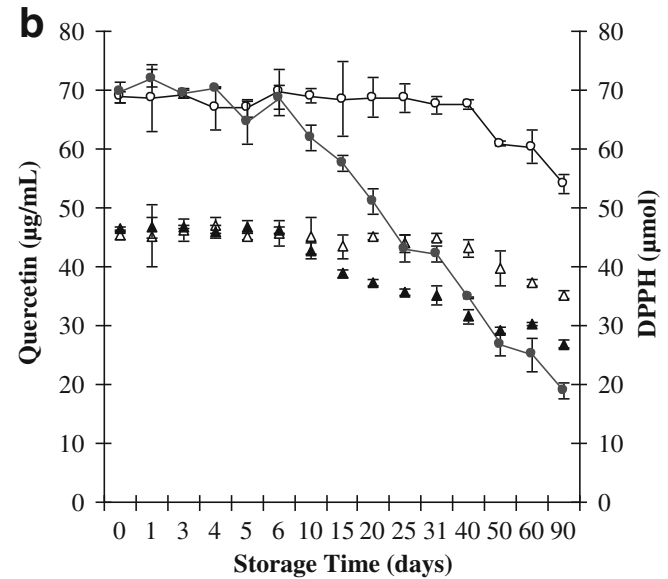

circles concentration of Quercetin-Np stored at $30{ }^{\circ} \mathrm{C}$, white triangles DPPH scavenging capacity by Quercetin-Np stored at $4{ }^{\circ} \mathrm{C}$, and black triangles DPPH scavenging capacity by Quercetin-Np stored at $30^{\circ} \mathrm{C}$. All determinations were performed in triplicate and the results expressed as mean \pm standard deviation 
degradation (oxidation) of quercetin present in the Np's. This phenomenon can be explained by the fact that lecithin, the main constituent of Np's, consists of unsaturated phospholipids susceptible to spontaneous oxidation by molecular oxygen dissolved in the suspension. This hypothesis was confirmed by detection of peroxides in $\mathrm{Np}$ after 10 days of storage at $30{ }^{\circ} \mathrm{C}$ (data not shown). Storage at $4{ }^{\circ} \mathrm{C}$ reduced the rate of oxidation of Quercetin-Np and quercetin degradation started to be observed after the 50th day of storage.

\section{Conclusion}

Quercetin has been successfully encapsulated in lecithin/ chitosan Np's using the self-assembly technique. This study showed that Quercetin-Np is stable to temperature (5 to $70{ }^{\circ} \mathrm{C}$ ) and $\mathrm{pH}$ variations in values ranging between 3.3 and 5.0. NP's stored at $4{ }^{\circ} \mathrm{C}$ showed resistance to aggregation for 90 days and stability to retention of quercetin concentration for 40 days. The results suggest that Quercetin-Np can be used as an ingredient of functional foods or as an alternative in the defense against free radicals damaging foods in hydrophilic environments. The features presented by Quercetin-Np make them attractive not only for the food industry, but also for cosmetic and pharmaceutical industries.

Acknowledgments Author Marthyna Pessoa de Souza thanks Coordenação de Aperfeiçoamento de Pessoal de Nivel Superior (CAPES/PDEE-Brazil) and Fundação de Amparo à Ciência e Tecnologia do Estado de Pernambuco (FACEPE, Brazil) for granting her scholarships. Miguel A. Cerqueira is recipient of a fellowship from the Fundação para a Ciência e Tecnologia (SFRH/BPD/72753/2010, FCT, POPH-QREN, and FSE Portugal). This research was financially supported by research grants and fellowships from the Conselho Nacional de Desenvolvimento Científico e Tecnológico (CNPq), as well as the Coordenação de Aperfeiçoamento de Pessoal de Nível Superior (CAPES) and Fundação de Amparo à Ciência e Tecnologia do Estado de Pernambuco (FACEPE).

The support of EU Cost Actions FA0904 and FA1001 is gratefully acknowledged.

\section{References}

An, D., Zhang, Q., Wub, S., Wei, J., Yang, J., Dong, F., et al. (2010). Changes of metabolic profiles in urine after oral administration of quercetin in rats. Food and Chemical Toxicology, 48, 1521-1527.

Barbieri, S., Sonvico, F., Como, C., Colombo, G., Zani, F., Buttini, F., et al. (2013). Lecithin/chitosan controlled release nanopreparations of tamoxifen citrate: loading, enzyme-trigger release and cell uptake. Journal of Controlled Release, 167, 276-283.

Benzie, I. F. F., \& Strain, J. J. (1996). The ferric reducing ability of plasma (FRAP) as a measure of "antioxidant power": the FRAP assay. Analytical Biochemistry, 239, 70-76.

Carneiro-da-Cunha, M. G., Cerqueira, M. A., Souza, B. W. S., Teixeira, J. A., \& Vicente, A. A. (2011). Influence of concentration, ionic strength and $\mathrm{pH}$ on zeta potential and mean hydrodynamic diameter of edible polysaccharide solutions envisaged for multinanolayered films production. Carbohydrate Polymers, 85, 522-528.

Chitkara, D., Nikalaje, S. K., Mittal, A., Chand, M., \& Kumar, N. (2012). Development of quercetin nanoformulation and in vivo evaluation using streptozotocin induced diabetic rat model. Drug Delivery and Translational Research, 2, 112-123.

Chuah, A. M., Kuroiwa, T., Kobayashi, I., \& Nakajima, M. (2009). Effect of chitosan on the stability and properties of modified lecithin stabilized oil-in-water monodisperse emulsion prepared by microchannel emulsification. Food Hydrocolloids, 23, 600 610.

Dickinson, E. (2003). Hydrocolloids at interfaces and the influence on the properties of dispersed systems. Food Hydrocolloids, 17(1), 25-39.

Fang, Z., \& Bhandari, B. (2010). Encapsulation of polyphenols-a review. Trends in Food Science \& Technology, 21, 510-523.

Firuzi, O., Lacanna, A., Petrucci, R., Marrosu, G., \& Sasoa, L. (2005). Evaluation of the antioxidant activity of flavonoids by ferric reducing antioxidant powerQ assay and cyclic voltammetry. Biochimica et Biophysica Acta, 1721, 174-184.

Gerelli, Y., Bari, M. T. D., Deriu, A., Cantu, L., Colombo, P., Como, C., et al. (2008). Structure and organization of phospholipid/ polysaccharide nanoparticles. Journal of Physics: Condensed Matter, 20, 104211. 8pp.

Ghosh, S., Dungdung, S. R., Chowdhury, S. T., Mandal, A. K., Sarkar, S., Ghosh, D., et al. (2011). Encapsulation of the flavonoid quercetin with an arsenic chelator into nanocapsules enables the simultaneous delivery of hydrophobic and hydrophilic drugs with a synergistic effect against chronic arsenic accumulation and oxidative stress. Free Radical Biology \& Medicine, $51,1893-1902$.

Gramza, A., \& Korczak, J. (2005). Tea constituents (Camellia sinensis L.) as antioxidants in lipid systems. Trends in Food Science \& Technology, 16, 351-358.

Guzey, D., \& McClements, D. J. (2006). Formation, stability and properties of multilayer emulsions for application in the food industry. Advances in Colloid and Interface Science, 128(130), 227-248.

Hafner, A., Lovric, J., Voinovich, D., \& Filipovic-Grcica, J. (2009). Melatonin-loaded lecithin/chitosan nanoparticles: physicochemical characterisation and permeability through Caco-2 cell monolayers. International Journal of Pharmaceutics, 381, 205-213.

Heurtault, B., Saulnier, P., Pech, B., Proust, J. E., \& Benoit, J. P. (2003). Physico-chemical stability of colloidal lipid particles. Biomaterials, 24, 4283-4300.

Huang, Q., Yu, H., \& Ru, Q. (2010). Bioavailability and delivery of nutraceuticals using nanotechnology. Journal of Food Science, 75(1), R50-R57.

Kumari, A., Yadav, S. K., Pakade, Y. B., Singh, B., \& Yadav, S. C. (2010). Development of biodegradable nanoparticles for delivery of quercetin. Colloids and Surfaces. B, Biointerfaces, 80, 184192.

Li, H., Zhao, X., Ma, Y., Zhai, G., Li, L., \& Lou, H. (2009). Enhancement of gastrointestinal absorption of quercetin by solid lipid nanoparticles. Journal of Controlled Release, 133, 238-244.

Liu, J., Gong, T., Wang, C., Zhong, Z., \& Zhang, Z. (2007). Solid lipid nanoparticles loaded with insulin by sodium cholatephosphatidylcholine-based mixed micelles: preparation and characterization. International Journal of Pharmaceutics, 340, 153-162.

Magnuson, B. A., Jonaitis, T. S., \& Jeffrey, C. W. (2011). A brief review of the occurrence, use, and safety of food-related nanomaterials. Journal of Food Science, 76(6), R126-R133.

Panya, A., Laguerre, M., Lecomte, J., Villeneuve, P., Weiss, J., Mcclements, D. J., et al. (2010). Effects of chitosan and rosmarinate esters on the physical and oxidative stability of liposomes. Journal of Agricultural and Food Chemistry, 58, 5679-5684. 
Pool, H., Quintanar, D., Figueroa, J. D., Mano, C. M., Bechara, J. E. H., Godinez, L. A., et al. (2012). Antioxidant effects of quercetin and catechin encapsulated into PLGA nanoparticles. Journal of Nanomaterials, $124580,12 \mathrm{p}$.

Portes, E., Gardrat, C., Castellan, A., \& Coma, V. (2009). Environmentally friendly films based on chitosan and tetrahydrocurcuminoid derivatives exhibiting antibacterial and antioxidative properties. Carbohydrate Polymers, 76, 578-584.

Prashanth, K. V. H., \& Tharanathan, R. N. (2007). Chitin/chitosan: modifications and their unlimited application potential - an overview. Trends in Food Science \& Technology, 18, 117-131.

Ramalho, V. C., \& Jorge, N. (2006). Antioxidantes utilizados em óleos, gorduras e alimentos gordurosos. Química Nova, 29(4), 755-760.

Saija, A., Tomaino, A., Trombetta, D., Pellegrino, M. L., Titã, B., Messina, C., et al. (2003). 'In vitro' antioxidant and photoprotective properties and interaction with model membranes of three new quercetin esters. European Journal of Pharmaceutics and Biopharmaceutics, 56, 167-174.

Şenyiğit, T., Sonvico, F., Barbieri, S., Özer, O., Santi, P., \& Colombo, P. (2010). Lecithin/chitosan nanoparticles of clobetasol-17-propionate capable of accumulation in pig skin. Journal of Controlled Release, $142,368-373$

Shahidi, F., \& Abuzaytoun, R. (2005). Chitin, chitosan and co-products: chemistry, production, applications and health effects. Advances in Food and Nutrition Research, 49, 93-135.

Sonvico, F., Cagnani, A., Rossi, A., Motta, S., Di Bari, M. T., Cavatorta, F., et al. (2006). Formation of self-organized nanoparticles by lecithin/chitosan ionic interaction. International Journal of Pharmaceutics, 324, 67-73.

Timbola, A. K., Souza, C. D., Giacomelli, C., \& Spinelli, A. (2006). Electrochemical oxidation of quercetin in hydro-alcoholic solution. Journal of the Brazilian Chemical Society, 17(1), 139-148.

Wu, T.-H., Yen, F.-L., Lin, L.-T., Tsai, T.-R., Lin, C.-C., \& Chamd, T.M. (2008). Preparation, physicochemical characterization, and antioxidant effects of quercetin nanoparticles. International Journal of Pharmaceutics, 346, 160-168.

Zhang, Y., Yang, Y., Tang, K., Hu, X., \& Zou, G. (2008). Physicochemical characterization and antioxidant activity of quercetin-loaded chitosan nanoparticles. Journal of Applied Polymer Science, 107, 891-897. 